This item was submitted to Loughborough's Research Repository by the author.

Items in Figshare are protected by copyright, with all rights reserved, unless otherwise indicated.

\title{
Characterisation of ball impact conditions in professional tennis: matches played on hard court
}

PLEASE CITE THE PUBLISHED VERSION

http://dx.doi.org/10.1177/1754337115617580

\section{PUBLISHER}

Sage / @ IMechE

VERSION

AM (Accepted Manuscript)

\section{PUBLISHER STATEMENT}

This work is made available according to the conditions of the Creative Commons Attribution-NonCommercialNoDerivatives 4.0 International (CC BY-NC-ND 4.0) licence. Full details of this licence are available at: https://creativecommons.org/licenses/by-nc-nd/4.0/

\section{LICENCE}

CC BY-NC-ND 4.0

\section{REPOSITORY RECORD}

Lane, Benjamin D., Paul J. Sherratt, Xiao Hu, and Andy R. Harland. 2019. "Characterisation of Ball Impact Conditions in Professional Tennis: Matches Played on Hard Court". figshare. https://hdl.handle.net/2134/20077. 


\section{Characterisation of Ball Impact Conditions in Professional Tennis: Matches Played on Hard Court}

BEN LANE ${ }^{1,2,3}$, PAUL SHERRATT ${ }^{1}$, HU XIAO $^{3}$ \& ANDY HARLAND ${ }^{1}$

${ }^{1}$ Sports Technology Institute, Loughborough University, Leicestershire, LE11 3TU, U.K., ${ }^{2}$ Institute for Sports Research, Nanyang Technological University, 639798, Singapore, ${ }^{3}$ Materials Science \& Engineering, Nanyang Technological University, 639798, Singapore

Correspondence: Ben Lane, Sports Technology Institute, Loughborough University, Loughborough, Leicestershire, LE11 3TU, U.K.

E-mail: B.Lane@lboro.ac.uk

Keywords: Tennis, ball, impact, professional, velocity, angle 


\section{Abstract}

To assess ball performance for research and development purposes requires greater understanding of the impact conditions a tennis ball experiences in professional tournament play. Ball tracking information taken from three consecutive years of an ATP 250 tour event played on hard court were analysed. The frequency of first serves, second serves, racket impacts and surface impacts were assessed per game and extrapolated to show how many impacts a single ball is subjected to. Where applicable the pre- and post-impact velocity and angle were found and the distribution of each analysed. In total, data from 65 matches comprising 1,505 games were analysed. On average, each game contained 70.26 ( \pm 16.23 ) impacts, of which $9.23 \%, 3.16 \%, 37.78 \%$ and 49.83\% were first serves, second serves, racket impacts and surface impacts respectively. As a result, assuming all balls in play are used evenly, a single ball is expected to be subjected to $105( \pm 24)$ impacts over the course of the nine games that it is in play. The results of the investigation could be used to design a wear protocol capable of artificially wearing tennis balls in a way that is representative of professional play.

\section{Introduction}

The International Tennis Federation (ITF) is the governing body of tennis, regulating the rules of the game, including the tennis ball approval process. The number of balls in play at any one time, along with the ball change policy is at the discretion of the event organisers. Events on the men's and women's professional circuit (ATP World Tour, WTA events and Grand Slams) usually have six balls in play at any one time, with ball changes occurring after the first seven games and every nine games thereafter. The first set of balls is replaced two games earlier than subsequent sets to account for use in the pre-match warm up. Any ball used in any tournament played according to the Rules of Tennis shall have been approved annually (according to the ITF Ball Approval Process) by the ITF in an attempt to generate consistency in ball performance ${ }^{1}$.

It was noted in 2003 that the ITF did not specify any characteristics of the ball relating to durability in the approval process. ${ }^{2}$ As a result, the length of time an approved ball would remain within the laws of the game remained unknown. Subsequent studies followed to assess the degree to which tennis ball properties change with use and how to best replicate this change in the laboratory. ${ }^{3,4} \mathrm{~A}$ method was empirically derived that 
produced ball degradation comparable to the changes found in balls taken from real play; culminating in the introduction of the durability test in to the ITF ball approval process in 2009. The durability test involves the application of a wear regime to six randomly selected balls from a sample of 24 subjected to the approval process. The wear regime subjects each ball to 20 high velocity impacts and two minutes of artificial felt wearing. Post-wear regime results for mass, size, rebound and deformation are compared to the initial test results for the six balls in question and a maximum allowable change is defined. ${ }^{5}$

The purpose of the durability test is to assess if the ball in question is capable of withstanding nine games of elite level play. The ITF has deemed the test fit for purpose based on the aforementioned research into the properties of new, used, worn and unworn balls, as well as the different methods for replicating changes in the lab. The test is also simple, controlled and requires little manual input which suits the needs of the ITF. Changes in ball properties are deemed to match that of balls used in tournament play, with balls of the lowest quality failing as is sometimes the case in play. However, the test itself does not allow for the performance of a given ball to be determined, nor is it able to predict the expected life span of a tennis ball, which may be of interest to tennis brands and ball manufacturers. It is also not known to what extent the test is representative of what a ball typically endures during its nine games of use.

An alternative option of assessing ball longevity is player testing. Player testing is disadvantaged by a lack of control and the necessity to recruit players that are of the level required to scrutinise the ball sufficiently. Furthermore, it takes a significantly longer time to complete than laboratory tests.

To assess ball performance for research and development purposes requires a greater scientific understanding of the impact conditions a tennis ball experiences in tournament play. Consequently, it would be beneficial to understand the number of impacts a ball endures, in terms of surface impacts, racket impacts and serves; as well as the impact conditions, namely velocity, angle and spin. Characterising the game in this way may allow for the production of a more representative wear protocol capable of determining ball quality and life span. Moreover, analysing tennis in this way may be well suited to monitoring the nature of game over time and assessing if the game itself has fundamentally changed.

Similar studies have been conducted with the aim of measuring in play characteristics of impact scenarios in professional tennis. Most aimed to measure a specific set of parameters to more realistically represent a given scenario in the laboratory, ${ }^{6,7}$ whilst others aimed to measure a given parameter over time to assess if the nature of the game was changing. ${ }^{8,9}$ Choppin et al. ${ }^{7}$ measured ball and racket parameters at the 
2006 Wimbledon qualifying tournament in order to replicate typical values for a baseline top spin forehand shot in laboratory testing. A multiple high speed camera setup allowed for 3D analysis of the ball and racket within a $2 \mathrm{~m}^{3}$ capture volume. A single high speed camera system was utilised by Goodwill et al. ${ }^{8}$ and Kelley et al. ${ }^{9}$ in order to determine typical ball spin rates. Maximum spin rate for male serves was consistent between studies at around $4300 \mathrm{rpm}$ and results were comparable to Sakurai et $a .^{10}$ whereby the spin and spin axis of flat, slice and kick serves were analysed. Male top spin forehand shots on average produced $2300 \mathrm{rpm}$ (maximum 3800 $\mathrm{rpm}$ ) whilst female players have been shown to impart less spin on the ball than men. In terms of the frequency of different events, Mitchell et al. ${ }^{11}$ found an average of 30 shots were played per game when analysing 30 recreational standard university players. As a result, with 6 balls in play, any given ball is likely to be hit 45 times assuming all balls are used evenly. Further research utilising notational analysis techniques has been conducted with the aim of analysing tactical elements of the game $\mathrm{e}^{12-14}$ and physiological demands playing the game places on players. ${ }^{15-17}$ Johnson and McHugh ${ }^{15}$ analysed video recordings of certain players at the 2003 US Open, finding on average a total of $17.9( \pm 12.1)$ strokes per service game and $12.2( \pm 10.0)$ strokes per return game for the players in question. Rally length ${ }^{12}$, serve strategies ${ }^{13}$ and the effect of player handedness on ball placement strategy ${ }^{14}$ have warranted interest in literature.

The aim of this study was twofold; 1 ) to determine if the nature of the game had changed over the course of the three years of ATP 250 Thailand Open from 2011 to 2013. 2) Establish the number of impacts and relating parameters a ball endured during professional play to inform evidence-based tennis ball testing relating to wear and degradation. It was hypothesised that the nature of the game did not change over the three years.

\section{Method}

Ball tracking data from the ATP 250 Thailand Open Tour event over the course of three consecutive years from 2011 to 2013 were analysed. The data comprised 65 matches consisting of 1,505 games, spread across all rounds of the event including the semi-finals and final. Male professional players competed at the event, played on an indoor hard court surface using Dunlop Fort All Court tennis balls. Data were collected by the automatic linecalling system (Hawk-Eye Innovations Ltd., Basingstoke, UK) using a multi-camera setup and corresponding ball tracking software. The system is officially accredited by the ITF, having passed the automated line-calling 
evaluation, and is used as an officiating aid whereby players are able to challenge the call of the line judge should they disagree with a line call. The system has been reported to have a mean error of $3.6 \mathrm{~mm},{ }^{18,19}$ sufficient for the proposed aims of the study.



Figure 1: An example file containing ball tracking information for a single point

Each individual file contained ball tracking information for a single contested point, an example of which can be seen in Figure 1. The serve formed the start point of each file, and was determined through software on capture. The end of the file was determined by the operator terminating recording of the ball tracking information on conclusion of the point. As a result some of the racket and surface impacts analysed occur after the winner of the point had been determined.

The resulting files were processed using Matlab (Mathworks, Natick, USA), with each file split chronologically into blocks of information representing a single trajectory arc or surface contact. For example a typical file, whereby the ball is served and a return made by the opponent, the first block of information would represent the ball trajectory from serve to the ball making contact with the surface. The next block would contain information regarding the contact of the ball with the surface. The third block would represent the ball trajectory from end of bounce to impact with the returner's racket and the forth block of information representing the ball trajectory from racket impact to either the next surface contact or racket impact (in the case of a volley). 
Each trajectory arc within the point file was defined by a parametric equation between two points in time. The coefficients to a polynomial equation defining the trajectory were used to calculate the position of the ball in three dimensional space with respect to time. Values were calculated at time intervals of 0.001 seconds. Velocity and acceleration of the ball was then calculated by differentiating first the displacement and then the velocity with respect to time. As a result displacement, velocity and acceleration of the ball in the global $X, Y$ and $\mathrm{Z}$ axes (as defined in Figure 1) were calculated for each trajectory at a frequency of $1000 \mathrm{~Hz}$. The point files were grouped by match and the matches grouped by year, allowing each point to be analysed by match and by year. File nomenclature allowed the determination of the set, game and serve number $\left(1^{\text {st }}\right.$ or $\left.2^{\text {nd }}\right)$ of the corresponding point, consequently the number of games played in the match could be ascertained.

The trajectory information enabled the following impact scenarios to be identified: serve; pre-surface impact; post-surface impact; pre-racket impact and post-racket impact. Serves were identifiable as they represented the starting point of the first trajectory arc within the point file. Pre- and post-surface impacts were identifiable from the information given in the file as they fell either side of surface contact information. Pre- and post-racket impact data were defined by two adjacent trajectory arcs, whereby the ball was not in contact with the surface, and the ball's Y-velocity changed sign. Checks were made to ensure the ball making contact with the net was not counted as racket impacts. This enabled the data to be split in terms of the type of impact (serve, racket impact \& surface impact) for further analysis.

The frequency of each impact event was noted and the velocity of the ball before and after impact with either a racket or surface was estimated using the calculated value at the relevant end point of the trajectory. The inbound and outbound angles on impact with the surface were calculated using the dot product of the surface normal and the resultant velocity vector (taken from the end point of the trajectory arc). The angle measured being the angle between the surface and the resultant velocity vector, as opposed to the angle between the resultant velocity vector and the surface normal. The change in velocity is defined as the postimpact value minus the corresponding pre-impact value. Once defined, racket impacts, surface impacts and serves could be analysed independently. 


\section{Statistical Analysis}

One-way ANOVA (impact frequency, $1^{\text {st }}$ serves $\& 2^{\text {nd }}$ serves) and Kruskal-Wallis (all other variables) nonparametric tests were used to compare year on year results. Pairwise comparisons for statistically significant results were conducted using Tukey's HSD (honest significant difference) for ANOVA tests and the DunnBonferroni approach for Kruskal-Wallis tests. All tests were conducted at a 95\% confidence level $(P \leq 0.05)$.

Results

\section{Frequency of Impacts}

Table 1 provides a breakdown of the data set including the number of matches that were analysed and the number of impact events that occurred. The 1,505 games analysed consisted of over 12,000 serves, 40,000 racket impacts and 50,000 surface impacts. The mean number of games, serves, racket impacts and surface impacts per match were not found to vary significantly year on year (games per match $F(2,62)=0.201, P=.819$; $1^{\text {st }}$ serves per match $F(2,62)=0.162, P=.851 ; 2^{\text {nd }}$ serves per match $F(2,62)=0.021, P=.979 ;$ racket impacts per match $F(2,62)=1.169, P=.288 ;$ surface impacts per match $F(2,62)=1.168, P=.318)$.

Table 1: Tournament totals (mean per match \pm SD) for each year of the event

\begin{tabular}{|l|r|r|r|r|}
\hline Year & \multicolumn{1}{|c|}{$\mathbf{2 0 1 1}$} & $\mathbf{2 0 1 2}$ & $\mathbf{2 0 1 3}$ & Total \\
\hline Matches & 23 & 23 & 19 & 65 \\
\hline \multirow{2}{*}{ Games } & 536 & 543 & 426 & 1,505 \\
& $(23.3 \pm 6.2)$ & $(23.6 \pm 6.0)$ & $(22.4 \pm 6.5)$ & $(23.2 \pm 6.1)$ \\
\hline \multirow{2}{*}{$\mathbf{1}^{\text {st }}$ Serves } & 3,478 & 3,537 & 2,775 & 9,790 \\
& $(151.2 \pm 45.2)$ & $(153.8 \pm 39.5)$ & $(146.1 \pm 48.4)$ & $(150.6 \pm 43.7)$ \\
\hline \multirow{2}{*}{$\mathbf{2}^{\text {nd }}$ Serve } & 1,201 & 1,177 & 989 & 3,367 \\
& $(52.2 \pm 20.3)$ & $(51.2 \pm 13.9)$ & $(52.1 \pm 20.8)$ & $(51.8 \pm 18.2)$ \\
\hline \multirow{2}{*}{ Racket Impacts } & 14,351 & 15,335 & 10,382 & 40,068 \\
& $(624.0 \pm 252.3)$ & $(666.7 \pm 196.6)$ & $(546.4 \pm 285.6)$ & $(616.4 \pm 246.3)$ \\
\hline \multirow{2}{*}{ Surface Impacts } & 18,981 & 19,999 & 13,878 & 52,858 \\
& $(825.3 \pm 311.4)$ & $(869.5 \pm 242.5)$ & $(730.4 \pm 337.7)$ & $(813.2 \pm 298.1)$ \\
\hline
\end{tabular}

The mean frequency of each impact event that occurred per game and as a percentage of all impact events is given (Table 2). Almost $50 \%$ of the impacts were surface impacts, with racket impacts and serves accounting for 
$37.78 \%$ and $12.39 \%$ respectively. In total, an average of 70.26 impacts occurred per game, resulting in average of 632.31 for every nine games played.

Table 2: Mean (SD) frequency and percentage of total for each impact scenario

\begin{tabular}{|c|c|c|c|c|c|c|c|c|}
\hline \multirow[b]{2}{*}{ Event } & \multicolumn{4}{|c|}{ Mean Frequency per Game } & \multicolumn{4}{|c|}{$\%$ of Total } \\
\hline & 2011 & 2012 & 2013 & $\begin{array}{c}\text { Mean } \\
\text { (SD) }\end{array}$ & 2011 & 2012 & 2013 & Mean \\
\hline $1^{\text {st }}$ Serve & $\begin{array}{c}6.45 \\
(0.52)\end{array}$ & $\begin{array}{c}6.52 \\
(0.52)\end{array}$ & $\begin{array}{c}6.49 \\
(0.74)\end{array}$ & $\begin{array}{c}6.49 \\
(0.58)\end{array}$ & 9.22 & 8.70 & 9.99 & 9.23 \\
\hline $2^{\text {nd }}$ Serve & $\begin{array}{c}2.19 \\
(0.41) \\
\end{array}$ & $\begin{array}{c}2.19 \\
(0.39) \\
\end{array}$ & $\begin{array}{c}2.30 \\
(0.51) \\
\end{array}$ & $\begin{array}{c}2.22 \\
(0.43)\end{array}$ & 3.13 & 2.92 & 3.54 & 3.16 \\
\hline Racket Impacts & $\begin{array}{l}26.36 \\
(6.10)\end{array}$ & $\begin{array}{l}28.86 \\
(7.99)\end{array}$ & $\begin{array}{l}23.95 \\
(7.92)\end{array}$ & $\begin{array}{l}26.54 \\
(7.50)\end{array}$ & 37.71 & 38.46 & 36.91 & 37.78 \\
\hline Surface Impacts & $\begin{array}{l}34.91 \\
(6.74)\end{array}$ & $\begin{array}{l}37.46 \\
(8.72)\end{array}$ & $\begin{array}{l}32.16 \\
(8.76)\end{array}$ & $\begin{array}{l}35.01 \\
(8.24)\end{array}$ & 49.94 & 49.92 & 49.56 & 49.83 \\
\hline Total & $\begin{array}{c}69.90 \\
(13.13)\end{array}$ & $\begin{array}{c}75.03 \\
(17.19)\end{array}$ & $\begin{array}{c}64.90 \\
(17.46)\end{array}$ & $\begin{array}{c}70.26 \\
(16.23)\end{array}$ & 100 & 100 & 100 & 100 \\
\hline
\end{tabular}

Table 3 shows how the frequency of each event varies with percentage use. If all six balls in play are used evenly (16.67\% of the time) then a single ball would on average be subjected to 105 impacts, of which 53 would be impacts with the surface, 40 impacts with a racket and 13 serves (rounded to the nearest whole number). In comparison if a given ball is used half as much or twice as much as the mean it will be subjected to 53 and 211 impacts respectively.

Table 3: Number of impacts for a given ball for even (16.67\%), half (8.33\%) and double (33.33\%) use. Assuming six balls are in play for a period of nine games (mean (SD)).

\begin{tabular}{|l|r|r|r|r|r|}
\hline \% Use - 9 Games & \multicolumn{1}{|c|}{$\mathbf{1}^{\text {st }}$ Serves } & \multicolumn{1}{|l|}{$\mathbf{2}^{\text {nd }}$ Serves } & \multicolumn{1}{l|}{ Racket Impacts } & \multicolumn{1}{l|}{ Surface Impacts } & \multicolumn{1}{l|}{ Total } \\
\hline $\mathbf{8 . 3 3 \%}$ & $4.86(0.44)$ & $1.67(0.32)$ & $19.90(5.63)$ & $26.25(6.18)$ & $\mathbf{5 2 . 6 7}(\mathbf{1 2 . 1 7})$ \\
\hline $\mathbf{1 6 . 6 7 \%}$ & $9.73(0.88)$ & $3.33(0.65)$ & $39.82(11.26)$ & $52.52(12.37)$ & $\mathbf{1 0 5 . 4 1}(\mathbf{2 4 . 3 4 )}$ \\
\hline $\mathbf{3 3 . 3 3 \%}$ & $19.46(1.75)$ & $6.66(1.29)$ & $79.61(22.73)$ & $105.01(24.73)$ & $\mathbf{2 1 0 . 7 5 ( 4 8 . 6 8 )}$ \\
\hline
\end{tabular}

Serve Results: Ball Velocity

Characteristics of the $1^{\text {st }}$ and $2^{\text {nd }}$ serves are highlighted in Figure 2 . The mean velocity for all years combined was $51.96( \pm 4.64) \mathrm{m} \cdot \mathrm{s}^{-1}$ and $42.06( \pm 4.37) \mathrm{m} \cdot \mathrm{s}^{-1}$ for $1^{\text {st }}$ and $2^{\text {nd }}$ serves respectively. Statistically significant 
differences were found between years for both $1^{\text {st }}$ serves $(F(2,9,787)=101.406, P=.000)$ and $2^{\text {nd }} \operatorname{serves}(F(2$, $3,364)=36.974, P=.000)$. In both cases post-hoc results revealed differences between 2013 and both $2011\left(1^{\text {st }}\right.$ serve $P=.000 ; 2^{\text {nd }}$ serve $\left.P=.000\right)$ and $2012\left(1^{\text {st }}\right.$ serve $P=.000 ; 2^{\text {nd }}$ serve $\left.P=.000\right)$. This was not the case when comparing 2011 to $2012\left(1^{\text {st }}\right.$ serve $P=.626 ; 2^{\text {nd }}$ serve $\left.P=.564\right)$.

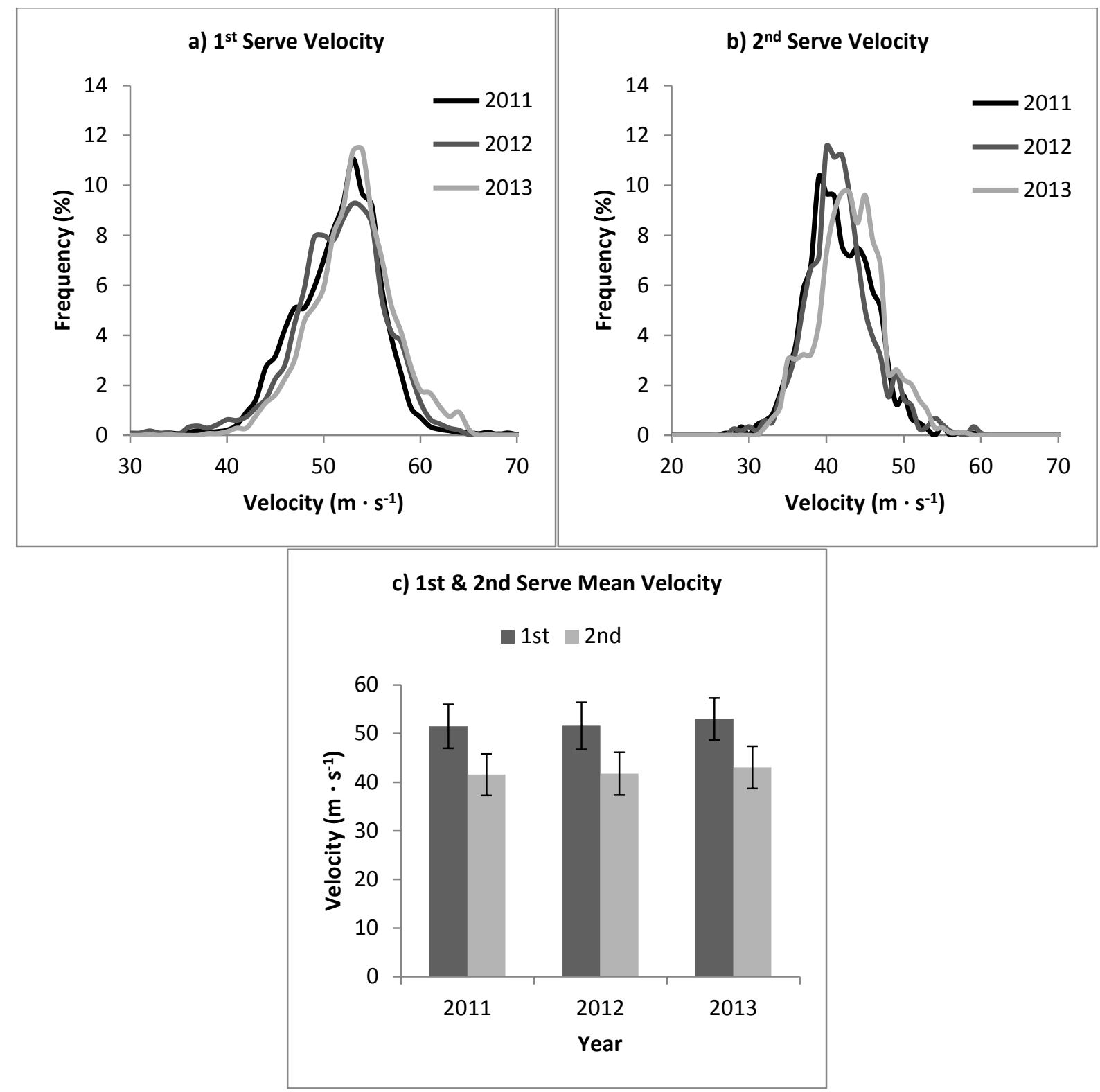

Figure 2: Frequency distributions for $1^{\text {st }}(\mathrm{a})$ and $2^{\text {nd }}(\mathrm{b})$ serve velocity and mean serve velocity \pm one standard deviation (c) 


\section{Racket Impact Results: Ball Velocity}

Ball velocity pre- and post-racket impact followed a bimodal distribution, as was the case with change in velocity

(Figure 3). With all of the distributions one peak is distinctly larger. The mean velocity pre-impact was 12.60 ( \pm 4.45) $\mathrm{m} \cdot \mathrm{s}^{-1}$ compared to a mean post-impact velocity of $30.59( \pm 6.29) \mathrm{m} \cdot \mathrm{s}^{-1}$, the ball velocity on average increased by $17.99( \pm 9.05) \mathrm{m} \cdot \mathrm{s}^{-1}$ due to impact with a players racket. Significant differences were found between all years for pre-impact ball velocity $\left(\chi^{2}(2)=227.960, P=.000 ; 2011-2012 P=.000 ; 2011-2013 P=.032\right.$; 2012-2013 $\mathrm{P}=.000)$, post-impact ball velocity $\left(\chi^{2}(2)=137.391, P=.000 ; 2011-2012 \mathrm{P}=.000 ; 2011-2013 \mathrm{P}=.003\right.$; $2012-2013 \mathrm{P}=.000)$ and change in velocity $\left(\chi^{2}(2)=236.166, P=.000 ; 2011-2012 \mathrm{P}=.000 ; 2011-2013 \mathrm{P}=.046\right.$; 2012-2013 $P=.000)$.




Figure 3: Frequency distribution for ball velocity pre-racket impact (a), post-racket impact (b) and change in velocity (c). Mean pre-, post- and change in velocity \pm one standard deviation (d).

\section{Surface Impact Results: Ball Velocity}

Figure 4 highlights the distribution of ball velocity pre-surface impact, velocity post-surface impact and relative change in velocity. Pre- and post-impact velocity followed a multimodal distribution whereas the change in velocity appears to resemble a bimodal distribution. The mean velocity of the ball across all years prior to impact with the surface was $21.64( \pm 7.99) \mathrm{m} \cdot \mathrm{s}^{-1}$, on average slowing due to contact by $6.69( \pm 2.07) \mathrm{m} \cdot \mathrm{s}^{-1}$, culminating in a mean post impact velocity of $14.96( \pm 6.34) \mathrm{m} \cdot \mathrm{s}^{-1}$. All variables showed statistically significant differences (pre-impact: $\chi^{2}(2)=34.733, P=.000$; post-impact: $\chi^{2}(2)=47.354, P=.000$ and change in velocity: $\chi^{2}(2)=234.324$, $P=.000)$. However, not all years differed from each other for pre-impact velocity $(2011-2012 P=.528 ; 2011-$ $2013 P=.000 ; 2012-2013 P=.000)$ and post-impact velocity $(2011-2012 P=.000 ; 2011-2013 P=.069 ; 2012-$ $2013 P=.000$ ). Post-hoc results revealed differences between all years for change in velocity (all comparisons $P$ $=.000)$. 


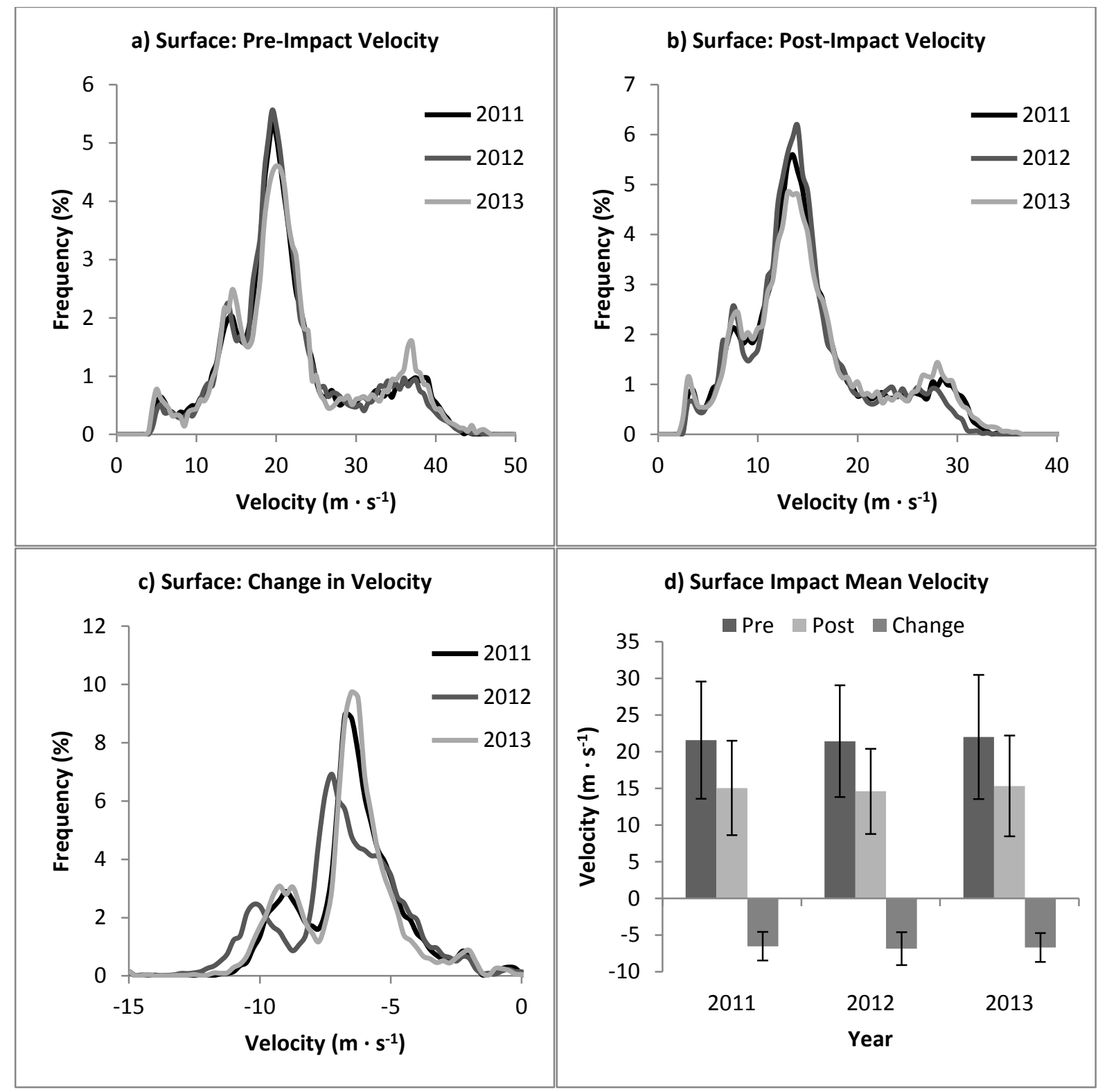

Figure 4: Frequency distribution for ball velocity pre-surface impact (a), post-surface impact (b) and change in velocity (c). Mean pre-, post- and change in velocity \pm one standard deviation (d).

\section{Surface Impact Results: Impact Angle}

The impact angle between the ball and the surface showed skewed distributions for both pre- and post-impact angle (Figure 5). The mean impact angles were $18.86^{\circ}( \pm 8.52)$ pre-impact and $23.08^{\circ}( \pm 9.18)$ post-impact. All variables showed statistically significant differences (pre-impact: $\chi^{2}(2)=49.144, P=.000 ;$ post-impact: $\chi^{2}(2)=$ 974.408, $\mathrm{P}=.000)$. Post-hoc results returned one insignificant result for pre-impact angle comparing 2011 and 
$2012(P=.769)$. All other paired comparisons for pre-impact and post-impact angle were significant whereby $P$ $=.000$.

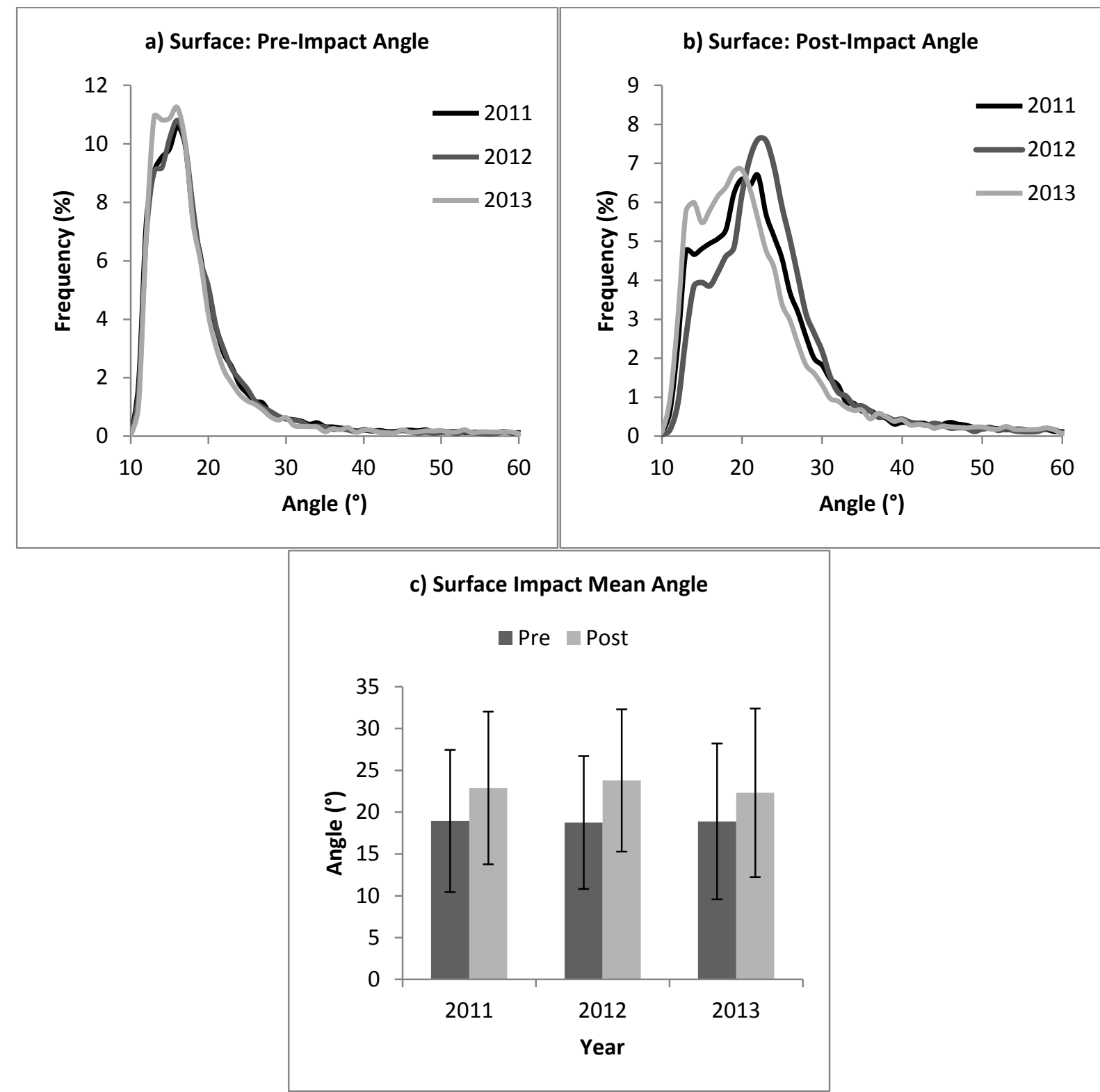

Figure 5: Frequency distribution for ball impact angle pre-surface impact (a), post-surface impact (b) and mean pre and post angle \pm one standard deviation (c).

\section{Discussion}

The aim of this study was twofold; 1 ) to determine if the nature of the game changed over the three years of data analysed. 2) To establish what a ball endures during professional play in terms of the number of impacts 
and corresponding impact parameters. Regarding the nature of the game the results of this investigation highlight how similar each year of the tournament is when assessed in terms of the frequency at which the given impact scenarios ( $1^{\text {st }}$ serves, $2^{\text {nd }}$ serves, racket impacts \& surface impacts) occur (Table 1$)$. This was evident whether assessing the tournament total, mean frequency per game or the percentage each type represented of the total number of impacts (Table 2). This was further highlighted by the percentage breakdown of each per game. In total serves, racket impacts and surface impacts account for $12.39 \%, 37.78 \%$ and $49.83 \%$ respectively, figures which were similar across the three years. The frequency of each impact event per match was not statistically significant when compared year on year (games per match $F(2,62)=0.201, P=.819 ; 1^{\text {st }}$ serves $F(2,62$ ) $=0.162, \mathrm{P}=.851 ; 2^{\text {nd }}$ serves $F(2,62)=0.021, \mathrm{P}=.979 ;$ racket impacts $F(2,62)=1.169, \mathrm{P}=.288$; surface impacts $F(2,62)=1.168, P=.318)$. This would indicate that the nature of the game, with respect to impact frequencies, did not significantly change over the course of the three years.

The mean number of racket impacts per game ( 26.54 excluding serves, 35.25 including serves) is comparable to a mean of 30 shots per game found by Mitchell ${ }^{11}$ and the mean combined total for strokes per service and return games (30.1) from the US Open ${ }^{15}$. The difference in standard of the players used by Mitchell is notable however (university against professional tour players).

The number of first serves relative to second serves compares favourably to statistics from the ATP Tour. The top 50 players on the ATP tour ranked in terms of first serve percentage for matches played on hard court ranged from $57-70 \%$ in $2011,55-71 \%$ for 2012 and $56-68 \%$ for $2013 .{ }^{20-22}$ At the lower end of the range it is expected that there are roughly twice as many first serves to second serves, as only around half of first serves are successful. At the higher end, the number of second serves is closer to one third the total number of first serves. In total for all years of the tournament 9,790 first serves and 3,367 second serves were analysed, indicating 6,423 first serves were successful. This equates to a first serve percentage of $65.6 \%$, within the tour range for the years in question and similar to Gillet et al. ${ }^{13}$ who reported a mean first serve percentage of $62 \%$ from matches played at the French Open (clay court) from 2005-2006. Hornery et al. ${ }^{17}$ reported slightly lower values from professional players of $56 \%$ on hard court and $55 \%$ on clay court.

When considering a single ball throughout its nine game lifespan a mean total of $105( \pm 24)$ impacts were calculated (Table 3), far more than the 20 high velocity impacts a ball is subjected to during the ITF durability test in the ball approval proves. Consequently, it is deemed unrepresentative of what a ball endures during professional play even though the test itself may produce similar levels of wear in terms of core softening and 
felt loss. ${ }^{4}$ The mean number of racket impacts (53 including serves) was also higher than the number of strokes reported by Mitchell et al. ${ }^{11}(45)$ for a given ball in play for nine games.

When considering ball velocity and impact angle, all corresponding statistical tests comparing year on year serve velocity $\left(1^{\text {st }}: F(2,9,787)=101.406\right.$ and $\left.2^{\text {nd. }}: F(2,3,364)=36.974\right)$, ball velocity during racket impacts $\left(\right.$ pre: $\chi^{2}(2)=$ 227.960, post: $\chi^{2}(2)=137.391$ and change: $\left.\chi^{2}(2)=236.166\right)$ and ball velocity and angle during surface impacts (velocity pre: $\chi^{2}(2)=34.733$, post: $\chi^{2}(2)=47.354$ and change: $\chi^{2}(2)=234.324$; angle pre: $\chi^{2}(2)=49.144$ and post: $\chi^{2}(2)=974.408$ ) returned a $P=.000$ result. Although not all post-hoc tests were also significantly different, these results clearly suggest that impact velocities and angles were not the same year on year, perhaps indicating that the nature of the game had changed with respect to these variables, even if the impact frequencies could be considered the same. Although the statistical tests indicate the variables were different, it is difficult to determine the significance of these differences in reality. Many of the distributions were complex, with multiple peaks, and at times relatively noisy data, which makes determining how different they are very difficult. Visually, most of the distributions appear very similar, following the similar trends.

Mean serve velocities of $51.96( \pm 4.64) \mathrm{m} \cdot \mathrm{s}^{-1}$ for $1^{\text {st }}$ serves and $42.06( \pm 4.37) \mathrm{m} \cdot \mathrm{s}^{-1}$ for $2^{\text {nd }}$ serves compares favourably to Hornery et al. ${ }^{17}$ who reported mean velocities of $49( \pm 2.5) \mathrm{m} \cdot \mathrm{s}^{-1}$ and $37.5( \pm 2.8) \mathrm{m} \cdot \mathrm{s}^{-}$ ${ }^{1}$ for first and second serves respectively. Both results show a drop in velocity of around $10 \mathrm{~m} \cdot \mathrm{s}^{-1}$ between first and second service velocity. This could be linked to the spin imparted on the ball by the players as Sakurai et al. ${ }^{10}$ reported a trade-off between velocity and spin for different types of serve (flat, slice and kick). Mean velocity was reported to decrease from $52.0 \mathrm{~m} \cdot \mathrm{s}^{-1}$ to $46.4 \mathrm{~m} \cdot \mathrm{s}^{-1}$ to $40.8 \mathrm{~m} \cdot \mathrm{s}^{-1}$, whilst spin increased from 1217 rpm to 2217 rpm to 3214 rpm for flat, slice and kick serves respectively. No significant difference was found when comparing 2011 and 2012 serve velocity $\left(1^{\text {st }}\right.$ serve $P=.626 ; 2^{\text {nd }}$ serve $\left.P=.564\right)$ whereas both were significantly different to $2013\left(2011: 1^{\text {st }}\right.$ serve $P=.000,2^{\text {nd }}$ serve $P=.000 ; 2012: 1^{\text {st }}$ serve $P=.000,2^{\text {nd }}$ serve $P=$ .000). This was due to a slight increase in 2013 mean serve velocity by approximately $1.5 \mathrm{~m} \cdot \mathrm{s}^{-1}$ compared to 2011 and $2012\left(1^{\text {st }}\right.$ serve mean $2011=51.50 \mathrm{~m} \cdot \mathrm{s}^{-1}, 2012=51.60 \mathrm{~m} \cdot \mathrm{s}^{-1}, 2013=53.01 \mathrm{~m} \cdot \mathrm{s}^{-1} ; 2^{\text {nd }}$ serve mean $2011=41.56 \mathrm{~m} \cdot \mathrm{s}^{-1}, 2012=41.74 \mathrm{~m} \cdot \mathrm{s}^{-1}, 2013=43.05 \mathrm{~m} \cdot \mathrm{s}^{-1}$ ) (Figure $2 \mathrm{c}$ ).

Choppin et al. $^{7}$ found an average ball velocity pre- and post-racket impact of $9.7 \mathrm{~m} \cdot \mathrm{s}^{-1}$ and $33.9 \mathrm{~m} \cdot \mathrm{s}^{-}$ ${ }^{1}$ respectively, compared to $12.9 \mathrm{~m} \cdot \mathrm{s}^{-1}$ and $30.2 \mathrm{~m} \cdot \mathrm{s}^{-1}$ in this investigation. However, their data were collected during practice conditions on a grass court surface, resulting in a greater increase in ball velocity through impact 
of $24.2 \mathrm{~m} \cdot \mathrm{s}^{-1}$ compared to a mean change of $17.99 \mathrm{~m} \cdot \mathrm{s}^{-1}$ in this study. The high speed camera methodology employed by Choppin et al. ${ }^{7}$ also enabled the calculation of additional parameters including impact location, ball spin and racket velocity.

Surface impact parameters have received less attention than racket impacts and serves with no reported velocity or angle results taken directly from professional tournament play. Spin off the ground has received the most attention with mean spin post-surface impact on grass courts reported in the region of 3,100-3,300 rpm..$^{8,9}$ Results from this investigation concluded on average the ball approached the surface with a mean velocity of $21.64( \pm 7.99) \mathrm{m} \cdot \mathrm{s}^{-1}$, at an angle of $18.86^{\circ}( \pm 8.52)$ from the surface. The ball left the surface with a mean velocity of $14.96( \pm 6.34) \mathrm{m} \cdot \mathrm{s}^{-1}$ at an angle of $23.08^{\circ}( \pm 9.18)$, which resulted in a mean reduction in velocity of 6.69 $( \pm 2.07) \mathrm{m} \cdot \mathrm{s}^{-1}$.

A limitation of the study was the data set analysed was specific to one tournament, played in the same location over the course of three consecutive years. To determine if the results found are specific to this event the data could be compared to additional professional hard court events which may highlight if the results found are typical of the given surface. A particular area of interest would be to compare data across the three major surfaces used in professional tennis; hard court, grass and clay, to assess how the nature of the game varies with playing surface and what effect, if any, this equates to with respect to the ball. As an extension of this work, future research should also look to investigate relevant factors that could not be directly obtained from the ball tracking data in this study, such as racket velocity, racket angle, the ball's impact location on the racket strings and ball spin.

Overall the results of this study would appear to indicate that the nature of the game of tennis at the ATP Thailand Open from 2011-2013 did not change. Year on year impact frequencies for serves, racket impacts and surface impacts indicate that the average composition of a game did not change. Although statistical differences were evident between years for ball velocity and angle, the differences appear very small suggesting some variation rather than a distinct change in the way the game was played. A number of factors could have compounded to produce this variation from the individuals involved each year (and how many games each were involved in), variation in equipment (ball rubber compound, surface, rackets and strings used) and variation in climatic conditions. 
The results of this investigation also enable the development of a more representative artificial ball wearing protocol, providing the foundations for replicating play in the lab. The frequency of which the ball endures impacts with the racket and the surface has been found and can be replicated. Using the percentage breakdown of the different impact scenarios and the corresponding velocity distributions in this investigation a ball could be artificially worn or degraded in a controlled and representative manner. Assuming the data in this study are representative of an average game, a tennis ball could be tested to failure to determine its life span (as a number of games).

\section{Conclusion}

The impact conditions of ball-surface and ball-racket impacts have been characterised to some extent although the characterisation of additional parameters, such as spin and racket velocity, would provide a more complete picture of what the ball endures during a professional tennis match. In order to determine if these results are typical of what occurs both within and between surfaces, further analysis of other tournaments would be required. The results however could be used to design a wear protocol capable of artificially wearing tennis balls in a more representative way to that of their intended use than the ITF durability test. As a result it may be possible to design a wear-protocol capable of comparing ball quality, both within a sample of the same ball or between ball types.

\section{Acknowledgement}

The authors would like to thank Hawk-Eye Innovations for allowing access to the data. No funding was received to conduct this research. 


\section{References}

1. ITF. ITF Ball Approval, http://www.itftennis.com/technical/apply/ball-approval/itf-ball-approval.aspx (2015, accessed 13 August 2015).

2. Miller S, Messner S. On the dynamic coefficient of restitution of tennis balls. In: Miller S (ed) Tennis Science \& Technology 2. Roehampton: ITF Licensing (UK) Ltd, 2003, pp. 97-104.

3. Capel-Davies J, Miller S. Durability of tennis balls worn in a test rig. In: Miller S (ed) Tennis Science \& Technology 2. Roehampton: ITF Licensing (UK) Ltd, 2003, pp. 113-122.

4. Spurr J, Capel-Davies J. Tennis ball durability: simulation of real play in the laboratory. In: Miller S, Capel-Davies J (eds) Tennis Science \& Technology 3. Roehampton: ITF Licensing (UK) Ltd, 2007, pp. 4148.

5. ITF. ITF Approved Tennis Balls, Classified Surfaces \& Recognised Courts. 2014.

6. Choppin S, Goodwill S, Haake S, et al. Ball and Racket Movements Recorded at the 2006 Wimbledon Qualifying Tournament. In: Estivalet M, Brisson P (eds) The Engineering of Sport 7. Paris: Springer Verlag, 2009, pp. 536-542.

7. Choppin S, Goodwill S, Haake S. Impact characteristics of the ball and racket during play at the Wimbledon qualifying tournament. Sport Eng; 13: 163-170, http://link.springer.com/10.1007/s12283011-0062-7 (2011, accessed 9 October 2013).

8. Goodwill S, Capel-Davies J, Haake S, et al. Ball spin generation by elite players during match play. In: Miller S, Capel-Davies J (eds) Tennis Science \& Technology 3. Roehampton: ITF Licensing (UK) Ltd, 2007, pp. 349-356.

9. Kelley J, Goodwill S, Capel-Davies J, et al. Ball Spin Generation at the 2007 Wimbledon Qualifying Tournament. In: Estivalet M, Brisson P (eds) The Engineering of Sport 7. Paris: Springer Verlag, 2009, pp. 543-550.

10. Sakurai S, Reid M, Elliott B. Ball spin in the tennis serve: spin rate and axis of rotation. Sport Biomech 2013; 12: 23-29.

11. Mitchell S, Caine M, Bowyer S. Playing with a larger tennis ball 2: A validation study on the effects on point play, player perception, muscle fatigue and soreness. Loughborough University, 2000.

12. O' Donoghue P, Ingram B. A notational analysis of elite tennis strategy. J Sports Sci 2001; 19: $107-115$.

13. Gillet $E$, Leroy $D$, Thouvarecq $R$, et al. A notational analysis of elite tennis serve and serve-return strategies on slow surface. J strength Cond Res 2009; 23: 532-539.

14. Loffing F, Hagemann N, Strauss B. Automated processes in tennis: do left-handed players benefit from the tactical preferences of their opponents? J Sports Sci 2010; 28: 435-443.

15. Johnson CD, McHugh MP. Performance demands of professional male tennis players. Br J Sports Med 2006; 40: 696-699.

16. Fernandez J, Mendez-Villanueva A, Pluim BM. Intensity of tennis match play. Br J Sports Med 2006; 40: 387-391. 
17. Hornery DJ, Farrow D, Mujika I, et al. An integrated physiological and performance profile of professional tennis. Br J Sports Med 2007; 41: 531-536.

18. Hawk-Eye Innovations. Hawk-Eye's Accuracy \& Reliability: Electronic Line Calling.

19. Collins HM, Evans RJ. You cannot be serious! Public understanding of technology with special reference to 'Hawk-Eye'. Public Underst Sci; 17: 283-308, http://dx.doi.org/10.1177/0963662508093370 (2008).

20. ATP World Tour. Stats Centre: 2011 1st Serve on Hard From All Countries, http://www.atpworldtour.com/en/stats/1st-serve/2011/hard/all/ (2015, accessed 5 August 2015).

21. ATP World Tour. Stats Centre: 2012 1st Serve on Hard From All Countries, http://www.atpworldtour.com/en/stats/1st-serve/2012/hard/all/ (2015, accessed 5 August 2015).

22. ATP World Tour. Stats Centre: 2013 1st Serve on Hard From All Countries, http://www.atpworldtour.com/en/stats/1st-serve/2013/hard/all/ (2015, accessed 5 August 2015). 